\title{
Las elecciones siguen siendo cosa de dos
}

Desde hace tiempo, venimos advirtiendo que el país respiraría elecciones durante el ciclo electoral que comenzó con las elecciones municipales y legislativas de 2012, continuaría con las presidenciales de 2014 y terminará con otras elecciones municipales y legislativas en marzo de 2015. En los últimos meses de 2013 y en estos inicios de 2014, confirmamos la omnipresencia de lo electoral en la vida nacional. Prácticamente, no hay cosa que suceda, que se diga o que se haga, para bien o para mal, que no adquiera una connotación político-electoral. El ejercicio del poder que se ha hecho en el país a lo largo de nuestra incipiente democracia ha provocado que la política y los políticos adquieran el don de devaluar lo que tocan. Así, acciones o acontecimientos que en tiempo "normal" tendrían un significado son desvirtuados por los intereses electorales de quienes los hacen o son tergiversados por las lecturas ideológicas de quienes los interpretan. Ni la naturaleza se escapa de ser contaminada con la humareda de la campaña. El 29 de diciembre, el volcán Chaparrastique, en el departamento de San Miguel, hizo erupción explosiva, lanzando cenizas y levantando una columna de humo que, de acuerdo a las autoridades, alcanzó los cinco kilómetros de altura. En otro tiempo, este tipo de acontecimientos hubiera despertado la solidaridad del pueblo con los damnificados. Pero en esta ocasión, el accionar de los políticos partidarios promocionando ayudas con los colores de sus partidos, más interesados en salir en la foto que en ayudar, minó una posible y generosa colaboración del pueblo salvadoreño. Hasta el presidente de la República, como actor importante de la campaña, llegó a pedir que voten por el candidato que garantice la continuidad de los cambios iniciados por él. En tiempos de campaña, los políticos de oficio juegan con el dolor de la gente traficando electoralmente con sus necesidades.

El actual proceso electoral presenta algunas novedades objetivas con respecto al pasado. Por primera vez, se aplicará el voto residencial en todos los municipios del país, lo que se espera motive a los 4955107 salvadoreños y salvadoreñas inscritos en el padrón para estas elecciones. Por primera vez, salvadoreños podrán votar desde el exterior a través del correo tradicional. De más de tres millones de compatriotas que, según el Tribunal Supremo Electoral (TSE), viven fuera, solo la exigua suma de 10337 se inscribieron. Pero lo importante es que la brecha se ha abierto para tomar en cuenta a quienes mantienen la vida de sus familias y soportan la economía nacional. Y también por primera vez, tres candidatos presentaban posibilidades de ganar. El lanzamiento de Tony Saca como candidato presidencial en febrero de 2013 parecía convertir las elecciones, por primera vez desde que se firmaron los Acuerdos de Paz, en asunto de tres. Saca se lanzó acuerpado por la coalición UNIDAD, conformada por los históricos Democracia Cristiana, Conciliación Nacional y por la Gran Alianza por la Unidad Nacional, GANA, una 
escisión de Arena después de la derrota electoral del año 2009. Ya antes, las candidaturas de Salvador Sánchez Cerén, del FMLN, (anunciada a mediados de 2012) y del edil capitalino Norman Quijano, por ARENA, (anunciada en agosto del mismo año) habían comenzado una de las campañas más prolongadas en la época reciente. Los otros dos aspirantes, René Rodríguez Hurtado, del Partido Salvadoreño Progresista (PSP), y Óscar Lemus, de la Fraternidad Patriótica Salvadoreña (FPS), ambas agrupaciones conformadas para esta elección, prácticamente tienen nulas posibilidades de ganar los comicios y su presencia solo ha sido ornato para los otros contendientes.

\section{Campañas basadas en el pasado y en el presente...}

Más allá de las novedades anteriores, la campaña no se ha diferenciado mucho de las que hemos presenciado en otros eventos similares. Su devenir ha arrojado como principal novedad la posibilidad de que no haya una segunda vuelta, necesaria de acuerdo a la legislación del país cuando un candidato no alcanza el 50 \% más uno de los votos. Hace unos meses, sobre todo con la incursión de Antonio Saca como aspirante presidencial, una segunda vuelta era cosa segura para casi todo el mundo. Pero en las últimas semanas, varias encuestas, sobre todo las respaldadas por instituciones académicas, revelaron que la posibilidad de una victoria del FMLN en primera vuelta no hay que descartarla. A estas alturas del proceso electoral, es tan temerario afirmar a raja tabla que habrá segunda vuelta - recurso al que se aferra la derecha- como también lo es afirmar que no la habrá — deseo del FMLN-.

Lo que ha caracterizado la campaña, en lo que respecta a las tres candidaturas con posibilidades de ganar, es que cada partido o coalición se ha centrado en un pilar en torno al cual giró toda su estrategia. Así, Unidad ha apostado todo o casi todo a la experiencia y personalidad del expresidente Saca, resaltando los que consideran logros de su pasada gestión. La apuesta del FMLN gira alrededor de continuar los cambios iniciados por el actual Gobierno. Por el contrario, Arena centró toda su estrategia en desvirtuar la gestión del Gobierno de Mauricio Funes, apelando volver al pasado de los Gobiernos de Arena.

Lo que el largo y cansino proceso electoral ha dado de sí hasta el momento refleja que la candidatura de Antonio Saca no ha logrado romper la polarización política en la que se encuentra el país desde el conflicto armado. Saca se presentó como la opción intermedia entre las dos extremas, proclamándose como la única fuerza capaz de unir a todos los salvadoreños —de ahí el nombre de "unidad" - , pero su movimiento ni ha sido tan fuerte ni se ha portado como una verdadera coalición. El eslogan "Tony Saca es unidad" expresa fielmente la mayor tragedia de su movimiento, que consiste en que se agota en la figura del expresidente. La fortaleza en la que descansaba la coalición es la experiencia y la personalidad de Saca. Esta apuesta no solo fue del mismo expresidente deseoso de volver a ocupar la primera magistratura, sino también de otros actores nacionales. El logo adoptado por el Gobierno de Funes al cumplir cuatro años de su gestión, que contiene 
los tres colores de los tres partidos integrantes de la coalición que respalda a Saca, hizo pensar a algunos que también el presidente apostaba por Saca. Otros hechos, como por ejemplo la absoluta falta de crítica de Funes a la gestión de Saca - lo cual contrasta con sus reiterados señalamientos a los otros Gobiernos de Arena-, inclinan a pensar lo mismo.

La estrategia de Saca ha sido captar el voto de la derecha, relegando a un tercer lugar al partido que lo echó por la puerta de atrás. Los que lo respaldan asumieron con demasiada confianza que los votos de los partidos de derecha que soportan su coalición están seguros. La elección de Francisco Laínez, canciller durante la gestión de Saca, como su compañero de fórmula tenía la intención de conquistar a los areneros descontentos con la candidatura de Quijano. Pero la estrategia parece que se les ha venido abajo. La cuestión es que lo que parecía ser asunto de tres a inicios del año 2013 ha vuelto a ser ahora, como siempre, cosa de dos, al menos de acuerdo a todas las encuestas en el país que se presentaron a fines de 2013 y principios de 2014. De la campaña de Saca hay que decir que, en términos generales, ha sido una campaña positiva, en el sentido de enfocarse más en hacer promesas, acudiendo a lo que considera los logros de su gestión como presidente, que en atacar a sus contendientes. La principal virtud reivindicada por Saca - su experiencia- ha sido también su principal tropiezo. El ser una figura conocida ya por la población y los cuestionamientos a su administración lo han relegado a un casi seguro y lejano tercer lugar en la primera vuelta de las elecciones, imposibilitándolo de llegar a una segunda vuelta, en la que los pronósticos le favorecían más y, probablemente, imposibilitándolo también de forzar una segunda ronda entre Arena y el FMLN.

Arena es el único partido que centró su campaña en términos negativos apelando volver al pasado. Su eslogan "vamos a recuperar a El Salvador" transmitió esta apuesta de volver a su pasado como gobierno. Por esto, su campaña ha estado más teñida de ataques que de propuestas. Sus candidatos han puesto mayor empeño en señalar lo que consideran fallos del actual Gobierno que en resaltar lo que los podría hacer diferentes y mejores para la población. La estrategia de Arena, contrario a lo que pretendía Saca, es aglutinar todo el voto de la derecha para forzar una segunda vuelta. La inclusión de una figura de fuera del partido, el Dr. René Portillo Cuadra, como compañero de fórmula de Norman Quijano pretendía darle un aire renovador e incluyente a la fórmula arenera para captar votantes también de fuera del partido. Ya sea porque Portillo en vez de abrir a Arena a planteamientos más progresistas fue absorbido por los planteamientos más conservadores del partido, ya sea porque no se le ha dado el espacio suficiente para hacerlo, Portillo Cuadra, de acuerdo a las encuestas, no ha logrado sumarle al partido de derecha.

Aunque las descalifiquen cuando no les favorecen, las encuestas han hecho mella en Arena y les hicieron replantear su marketing publicitario. Desde que las intenciones de voto no les favorecen y el crecimiento del FMLN en las preferencias electorales comenzó a dibujar la posibilidad de hacer innecesaria una segunda vuelta, la estrategia cambió. 
Y la sola posibilidad, por incierta que sea, de que el FMLN gane en primera vuelta ha logrado aglutinar a los sectores de la derecha hasta ahora dispersos por las diversas posiciones ante la candidatura de Quijano. El cambio de estrategia ha consistido en que Arena ha dejado como principal y casi única bandera de campaña el tema de la seguridad. "Vota por la seguridad, vota por Arena" es el eslogan que ha sustituido a "vamos a recuperar a El Salvador". La tónica de la campaña de Arena no ha cambiado. Sigue ensañándose en lo que consideran fallos del Gobierno actual, y se centra en la acusación de haber pactado con las maras y no con la población. En esta recta final de la campaña, todos los actores que apoyan al partido afín a la gran empresa privada van dejando su poco disimulado anonimato y se van alineando públicamente ante un eventual segundo gobierno del FMLN. Los medios de comunicación más grandes del país, desde que las encuestas no le favorecen a Arena, han puesto toda la carne en el asador para contrarrestar la caída en las preferencias electorales. Coincidentemente con la campaña de Arena por resaltar la inseguridad, los medios visibilizan más que nunca los hechos delincuenciales, sobre todo los más violentos que, de acuerdo a la Policía Nacional Civil, han aumentado en las últimas semanas.

Por su parte, el FMLN tiene como piedra angular de su campaña la continuidad y profundización de los cambios iniciados por el Gobierno de Mauricio Funes, ahora reconocido como el primer Gobierno del FMLN. Hace un año, esta identificación no era posible. Los enfrentamientos de Mauricio Funes con el FMLN fueron de conocimiento público. El empeño de Funes por separar su gobierno del partido claudicó antes de los cuatro años. El devenir de la campaña los unió. Desde que el actual Gobierno cumplió su cuarto año de gestión, en junio de 2013, el eslogan del frente comenzó a ser "4 años de gobierno" y el eslogan del Gobierno dejó de ser "unir, crecer, incluir" sustituyéndose por "buenos cambios".

Al escoger a su candidato, el FMLN volvió su vista solamente para adentro del partido. La experiencia vivida con Mauricio Funes provocó que la dirigencia efemelenista decidiera escoger a alguien de su redil que aglutinara a los sectores internos. Por ello, depositó, en la figura del alcalde de Santa Tecla, Óscar Ortiz, la misión de apuntalar la discreta candidatura de Salvador Sánchez Cerén. De acuerdo a las encuestas, la apuesta del frente les está dando los resultados que esperaban. En efecto, durante toda la campaña, el FMLN ha sido el partido que más ha promocionado a "la fórmula" como propuesta presidencial, distanciándose del resto de ofertas que se decantan casi por completo por la figura presidencial.

La campaña del FMLN, a diferencia de las de Arena y de Unidad, se ha basado en el presente para desde ahí prometer profundizar lo que para ellos son logros del actual Gobierno. En general, el tono de la campaña del FMLN, como la de Saca, ha sido positivo, insistiendo más en lo bueno ya hecho y en lo que dicen que van a hacer. El FMLN defiende el juego limpio de la campaña ante los ataques de Arena. Lo que hay que decir es que, en realidad, su campaña tiene otros dos afluentes caudalosos. No necesita mayor explica- 
ción ni admite dudas fundamentadas que Alba Petróleos tiene una campaña paralela a favor del FMLN. Y también es una obviedad que el Gobierno de la República, sobre todo a través de la figura del presidente Funes, tiene una campaña permanente en dos dimensiones: promoviendo la necesidad de continuar con los cambios iniciados en su Gobierno y el ataque frontal a las tres primeras gestiones de Arena, excluyendo de esta campaña negativa al Gobierno que presidió Antonio Saca. El FMLN mantiene, ciertamente, una campaña de talante positivo, porque el presidente Funes se ha encargado de la crítica negativa a Arena. De hecho, en los últimos meses, la figura del presidente se ha desdibujado, poniéndose en abierta campaña a favor de la candidatura del FMLN. Dicen que, en las democracias modernas, el mayor elector es el presidente saliente. Si esto es cierto, el FMLN no tendría por qué preocuparse, pues el presidente Funes está jugando un papel que sería decisivo.

La probabilidad de una victoria en primera vuelta, si en el lado contrario ha provocado la unión de los ánimos dispersos de la derecha, en la izquierda ha sido como una inyección de energía para hacer un gran esfuerzo que permita lograrlo. Las figuras mejor valoradas por la población en el actual Gobierno han dejado sus despachos para trabajar abiertamente en la campaña a favor del FMLN. Destaca entre estas figuras la de la Primera Dama, Vanda Pignato, que ha dejado sus labores como titular de Inclusión Social para sumarse al concierto de los que piden continuar y profundizar los cambios.

A pesar de las diferencias anotadas arriba, las campañas de los tres aspirantes de las agrupaciones políticas que se presentaron con posibilidades de ganar comparten, además de su ambición de llegar al poder, el vicio de ofrecer lo imposible. En la recta final, la campaña se dibuja como una especie de competencia de ciencia ficción. Arena ofrece eliminar a las maras y que el dinero florecerá con su gestión. Unidad promete doblar el número de policías y sacar adelante a los sectores más excluidos del país. El FMLN asegura la extensión de los programas sociales y una serie de medidas que difícilmente se podrán realizar. Ni la crítica situación fiscal que vive el país ni la complejidad de los grandes problemas con raíces estructurales hacen posible que esas promesas tengan un asidero de realidad.

En medio de la campaña que vivimos, hay dos acontecimientos que merece la pena comentar, por su importancia en el devenir electoral.

\section{Expresidente Flores queda en evidencia}

De cara al proceso electoral, el escándalo del extravío de 10 millones de dólares durante el Gobierno del presidente Francisco Flores (1999-2004), contrario a lo que algunas opiniones afirmaron, pudo haber causado un fuerte golpe a la candidatura de Norman Quijano y ser causante, por lo menos en parte, de la caída en las preferencias electorales.

En el mes de noviembre, fue el presidente Funes el encargado de develar la sospecha. Flores descalificó la denuncia acusando de desvarío al presidente. Pero los sucesos que se dieron después revelaron que estamos ante un 
asunto que puso entre la espada y la pared al que se presentara como principal asesor de la campaña de Quijano. Que el presidente de la República, a pesar de su investidura, ha entrado en el juego de la campaña electoral se da por descontado, como lo hemos sostenido antes. Sería ingenuo pensar que sus actuaciones en este tema no tienen fines proselitistas. Pero los indicios del desvío de 10 millones de dólares no podían tomarse a la ligera y dejarse pasar, como suele suceder con la palabra de los políticos.

El punto neurálgico de este acontecimiento lo constituyó la comparecencia del expresidente Flores a una comisión de la Asamblea Legislativa formada sin la participación de diputados de Arena y con el fin expreso de investigar el caso. iError de cálculo! El presidente Flores fue a inculparse de recibir dinero de manera personal proveniente del entonces presidente de Taiwán, sin ningún mecanismo institucional que comprobara que ingresó a la hacienda pública ni una explicación convincente de su destino. Flores recibió ayuda directa del expresidente de Taiwán Chen Shui-bian, el mismo que purga una condena a cadena perpetua por corrupción y el mismo que envió "ayuda" al expresidente de Guatemala, Alfonso Portillo —-preso en los Estados Unidos por, entre otros delitos, desviar fondos provenientes de Taiwány al de Costa Rica, Miguel Ángel Rodríguez - acusado y encarcelado por la misma razón- La expresidenta panameña, Mireya Moscoso, también fue acusada en su país de recibir un millón de dólares como regalo de cumpleaños de parte del ahora encarcelado expresidente asiático.

Aunque Flores fue a confesar una serie de actuaciones que le pueden valer ser el primer expresidente enjuiciado del país, de cara al proceso electoral, su comparecencia, lejos de limpiar a Arena como piensan algunos que era su objetivo, perjudicó las aspiraciones del candidato arenero quien, en otro yerro político, lo confirmó como su asesor después de semejante declaración. Por supuesto que el FMLN está exprimiendo el caso con fines electorales. La comisión legislativa sigue "trabajando" y las declaraciones de Flores explicando el destino de los fondos han sido gradualmente desvirtuadas por todos los funcionarios de su Gobierno que han dado su declaración ante la comisión. El presidente Funes ha insistido en el tema hasta la saciedad extrapolando la actuación del Flores a todos los Gobiernos de Arena exceptuando, por supuesto, el de Antonio Saca.

\section{El debate que no fue debate}

Que no iba a ser debate se sabía desde que la Asociación Salvadoreña de Radiodifusores, ASDER, anunció el formato del evento del 12 de enero de ese año. En realidad, lo que vio el pueblo salvadoreño, en una masiva transmisión inédita por radio y televisión, fue el máximo consenso alcanzado por los partidos políticos. O si se prefiere, el máximo que la cultura política partidaria puede permitir hasta el momento.

El foro auspiciado por el Tribunal Supremo Electoral (TSE) fue promocionado como el "primer gran debate presidencial" por puras razones de impacto publicitario. 
La modalidad de la presente campaña, que ha seguido la tradición de la crítica destructiva y las promesas sin asidero, hacía que el evento se esperara como uno de los pocos espacios que permitieran ver reunidos a los cinco candidatos para hablar seriamente de lo que ofrecen al país. Pero, reconociendo el encomiable esfuerzo de ASDER y lo que significa el foro como precedente para futuras elecciones, el debate no arrojó novedades que no se hubieran escuchado ya en la kilométrica campaña electoral. Incluso aunque hubiese sido solo un foro como lo fue, el pueblo salvadoreño se merecía que los candidatos hablaran con más responsabilidad, compartiendo medidas concretas y posibles, y que demostraran un conocimiento profundo de la realidad nacional y una capacidad para comprenderla y mejorarla.

Pero el debate dejó en claro solo que las promesas de campaña se acercan más a cantos de sirenas que a proyectos realizables. Confirmó con mucha claridad las dotes de orador del expresidente Saca que, sin embargo, no le han rendido los réditos que esperaba. Y también dejó evidencia de la imposibilidad de los otros candidatos para granjearse la empatía emocional con el electorado. La nota del debate la puso el candidato de Arena, uno de los dos fuertes aspirantes a la presidencia, al revelar su carta para acabar con las maras en el país. Arena apuesta por la militarización de la seguridad pública y por la aplicación del Código de Justicia Militar, a los mareros, cuestión que puede ser interpretada como fusilamiento. Promesas también imposibles de implementar de acuerdo a la Constitución del país y que exigirían desandar el camino que abrieron los Acuerdos de Paz de 1992. Probablemente, esta propuesta despierte simpatía en un sector de la población, sobre todo en aquellas personas que están de acuerdo con el exterminio físico de los mareros. Pero también puede ser arma de doble filo por el rechazo que puede provocar en las personas que no quieren ver recrudecer la situación de violencia y creen que uno de los mayores aportes de los Acuerdos de Paz fue, precisamente, la separación de la seguridad pública de las funciones del Ejército. No obstante, la propuesta se enmarca dentro de la nueva estrategia de Arena de dejar como principal carta de su campaña la problemática de la inseguridad, focalizando el origen de esta en la juventud marera, sin tocar las otras estructuras del crimen organizado que se enquistan en poderosas e influyentes personas y estructuras de la sociedad salvadoreña.

Los últimos días de la campaña serán decisivos para que el FMLN confirme si no será necesaria una segunda vuelta o para que Arena la fuerce porque es la única posibilidad que tiene de disputar la presidencia. Una segunda vuelta nos pondría ante un escenario diferente en el que los pronósticos son reservados.

Mientras tanto, en esta cuenta retroactiva, el escenario que nos espera, si todo sigue su rumbo, es un recrudecimiento de las críticas destructivas de Arena al Gobierno del FMLN, acusándolo de incapacidad para gobernar y de tener como modelo a los Gobiernos de los países del ALBA. La inseguridad, por arte de magia, se disparará en estos últimos días y Arena levantará su mano dura para decir: "Nosotros tenemos la solución". Arena volverá a recurrir al recurso de generar miedo en la población, como lo hizo en otras 
ocasiones y que no le dio resultado en el 2009. Para este cierre de campaña, todas las fuerzas de derecha se aglutinarán en torno a la candidatura de Arena: profesionales, deportistas, instituciones y medios de comunicación harán a un lado la mesura y se vestirán con los colores que defienden.

Por su parte, el FMLN arreciará la campaña que exalta los logros del actual Gobierno y las promesas de mejorarlos. Y todos los que apoyan su proyecto también dejarán las butacas de la neutralidad y firmarán su apoyo, sobretodo para evitar la posibilidad de que regrese Arena al poder. El FMLN contará, por supuesto, con el decidido apoyo de las empresas Alba, que seguirán invirtiendo millonarios recursos con tal de evitar una segunda vuelta. Del Gobierno puede esperarse que siga desprestigiando a Arena y, a lo mejor, en los días venideros, puede de nuevo dar un campanazo haciendo público otro de los actos oscuros de los Gobiernos de Arena.

Antonio Saca tiene que comenzar a pensar en su actuación en cualquiera de los dos escenarios. Como la única posibilidad de ganar en primera vuelta es del FMLN, hipotéticamente en este escenario Saca habría ya adelantado un entendimiento. En una segunda vuelta entre el FMLN y Arena, Saca tendría que negociar el capital político que le dejen los partidos que conforman su coalición. Por lo visto, no sería mucho, pero le serviría para negociar apoyos y protecciones. Solo resta esperar el impacto de todo esto en los votantes, el 2 de febrero. 